\title{
Lehren für die Eurozone aus dem bundesdeutschen und US-amerikanischen Föderalismus
}

\author{
Christian Deubner*
}

\section{Solidarität, Kontrolle und ,moral hazard‘ in Deutschland, den USA und der Eurozone}

In Deutschland gibt es eine umfangreiche Literatur zu Fragen des deutschen Föderalismus, die durch die beiden Föderalismuskommissionen 2003-2004 und 2007-2009 noch einmal bereichert wurde. Im Hinblick auf die europäische Integration war die weit vorherrschende Meinung, dass die Deutschen ihr gegenüber besonders aufgeschlossen seien und auch besonders viel beizutragen hätten, weil sie aus den Erfahrungen mit dem eigenen Staatswesen schöpfen könnten.

Manche Erwartung wird bei einer genaueren Analyse allerdings enttäuscht. Ganz besonders bemerkbar und auch besonders bedauerlich ist dieser Mangel in der breiten Diskussion über die Konstruktion einer europäischen Fiskalunion, die seit Mitte 2010 rege geführt wird. Damit ist die Gründung einer Reihe von Koordinierungsinstitutionen der nationalen Finanzpolitiken und Fiskalpolitiken für die gesamte Eurozone gemeint sowie die massive Verstärkung der Gemeinschaftskompetenzen gegenüber den Eurostaaten mit Blick auf ihr fiskalpolitisches Verhalten, Regelsetzung, Überwachung, Koordinierung, bis hin zur Revision nationaler Politikentscheidungen. Stichworte sind hier: Six-Pack, Two-Pack, Fiskalpakt. ${ }^{1}$ Auf der anderen Seite stehen die sogenannten Rettungsschirme zur finanziellen Nothilfe für Eurostaaten und für Banken, der Europäische Finanzstabilisierungsmechanismus (EFSM), die Europäische Finanzstabilisierungsfazilität (EFSF) und der Europäische Stabilisierungsmechanismus (ESM), von denen ab 2013 nur noch der ESM weiter existieren wird, ${ }^{2}$ sowie der Einsatz der Europäischen Zentralbank als ,lender of last resort‘ für die Eurostaaten.

Der Beitrag, den deutsche Experten und Politiker aus ihren nationalen Erfahrungen an Forderungen, Erwartungen oder auch Bedenken in diese neuentstehende europäische Fiskalunion einbringen, ist sehr gering. ${ }^{3}$ Ähnlich ist das Ergebnis in Bezug auf andere föderale

* Dr. habil. Christian Deubner, Berlin.

Dieser Aufsatz basiert auf einem Vortrag zum selben Thema, den der Autor am 16. November 2012 auf dem Jahreskolloquium des Arbeitskreises Europäische Integration e.V. gehalten hat. Vgl. dazu Frédéric Krumbein: Für ein solidarisches und solides Europa, in diesem Band, S. 78-85. Der in diesem Beitrag enthaltene Vergleich konzentriert sich auf die Variablen Solidarität, Kontrolle und ,moral hazard" in der staatlichen Haushalts- und Fiskalpolitik. Um das tun zu können, mussten sehr bedeutsame andere Variablen ausgeblendet werden, darunter die Behandlung der Alt-Schulden oder ,legacy debts' der Eurostaaten und die Frage einer Banken-Union in der Europäischen Union, die für eine Gesamtbewertung der Reformen in der Eurozone ebenfalls von sehr großer Bedeutung sind.

1 Vgl. hierzu ausführlich Peter-Christian Müller-Graff: Editorial: Die rechtliche Neujustierung der Europäischen Wirtschafts- und Währungsunion, in: Zeitschrift für das gesamte Handelsrecht und Wirtschaftsrecht 176/2012, S. 2-14.

2 Vgl. Bundesfinanzministerium: Euro-Schutzschirm. Was sind EFSM, EFSF und ESM?, abrufbar unter: http:/ www.bundesregierung.de/Webs/Breg/DE/Themen/Euro/EFSFundESM/esm_efsm_efsf/_node.html (letzter Zugriff: 1.11.2012).

3 Eine der wenigen Ausnahmen, die beide Dimensionen, die deutsche und die europäische, ausdrücklich zusammenbringt, ist das in 2010 erstmals erschienene Buch von Kai Konrad/Holger Zschäpitz: Schulden ohne Sühne? Was Europas Krise uns Bürger kostet, München 2012. Die Lektüre dieses Buches hat bei der Klärung mancher Gedanken geholfen die im Folgenden vorgestellt werden, ohne dass dazu ausdrücklich auf einzelne Seiten oder Textstellen verwiesen werden könnte. 
Erfahrungen außerhalb Europas. Das ist erstaunlich und es ist auch sehr bedauerlich, denn für das Verständnis der krisenhaften Entwicklungen der Eurozone sind schon die deutschen Erfahrungen sehr instruktiv. Vor allem könnten sie helfen, in der Eurozone Fehler zu vermeiden, die beim Aufbau des bundesdeutschen Fiskalföderalismus gemacht und die bis heute noch nicht wirksam korrigiert wurden. Der internationale Vergleich könnte diese Perspektive noch deutlich weiten.

Der vorliegende Beitrag soll mit dazu beitragen, den nur aufEU-Konzepte und -Strukturen fokussierten Tunnelblick aufzugeben und die Öffnung hin zu den Erfahrungen mit dem Fiskalföderalismus der reifen Bundesstaaten weiter voranzutreiben.

\section{Bringt mehr Solidarität mehr fiskalpolitische Solidität und mehr Wachstum?}

In der aktuellen Schuldenkrise der Eurozone wird von Politikern und Experten mehr Solidarität für überschuldete Eurostaaten gefordert. Als Beispiele für solche Solidarität gelten Eurobonds, großzügigere Kredite zu geringeren Zinsen und leichterer Konditionalität. Ihr Zweck ist die Förderung von mehr Wachstum und autonomer Wirtschaftskraft, von dauerhafter Schuldentragfähigkeit und von langfristig ausgewogenen Wirtschaftsbeziehungen mit den Staaten des Nordens. Diese Forderung ist umstritten. Das Hauptgegenargument hat den Namen ,moral hazard', moralisches Risiko.

Der Begriff stammt aus der Versicherungswirtschaft und bezeichnet die dort gemachte Erfahrung, dass die gegen ein bestimmtes Schadensrisiko versicherten Personen oder Unternehmen vielfach dazu neigen, ihre eigenen Bemühungen zur Vermeidung dieses Schadens zu verringern beziehungsweise sich diesem Risiko eher auszusetzen als wenn sie selbst die Folgen des Schadens tragen müssten. ${ }^{4}$ Er wird inzwischen von Wissenschaft und Medien auf viele analoge Sachverhalte angewendet, also im Kontext staatlicher Verschuldung auch auf das Verhalten von Investoren und Schuldnern: Beispiele sind Investoren, die sich beim Vorhandensein eines solventen Bürgen für die von ihnen ausgegebenen Kredite wenig darum kümmern, wie solvent ihre eigenen Schuldner sind, und die Kredite nicht entsprechend dem tatsächlichen Risiko verteuern, sowie Schuldner, die sich im Bewusstsein der Bürgschaft und damit fortdauernd billiger Kredite leichtsinniger und höher verschulden als sie es sonst tun würden.

Die Befürchtung ist dann, dass ein Mehr an Solidarität gegenüber überschuldeten Eurostaaten gerade die Verhaltensweisen ermutigen könnte, die zu dieser Überschuldung geführt haben. Gegenüber diesen Befürchtungen wird vor allem von deutscher Seite eingewendet, dass eine wirkungsvolle europäische Kontrolle der mitgliedstaatlichen Fiskalpolitiken solche gefährlichen Tendenzen verhindern könnte. ${ }^{5}$

\section{Zwei reife Bundesstaaten als Beispiele des Fiskalföderalismus}

Zur weiteren Klärung dieser fiskalpolitischen Argumente werden im Folgenden zwei Typen reifer Bundesstaaten auf ihre spezifische Kombination von Solidarität und Kontrolle und auf ihre Ergebnisse bezüglich der fiskalpolitischen Stabilität ihrer Gliedstaaten getestet. Welcher Typus verhindert ,moral hazard‘ besser? Welche Elemente der jeweiligen Politik

4 Mathias Erlei: Moral Hazard, in: Wirtschaftslexikon Gabler, 2012, abrufbar unter: http://wirtschaftslexikon.gabler.de/Definition/moral-hazard.html\#referenzen (letzter Zugriff: 2.11.2012).

5 Vgl. Angela Merkel: Rede im Europäischen Parlament, 8.11.2012, abrufbar unter: http://www.bundeskanzlerin.de/Content/DE/Rede/2012/11/2012-11-07-merkel-eu.html?nn=74420 (letzter Zugriff: 10.12 2012). 
sind für das Ergebnis entscheidend? Welchem der beiden Typen ähnelt die entstehende europäische Fiskalunion? Können die Erfolgschancen der entstehenden Fiskalunion vor diesem Hintergrund bewertet werden?

\section{Bundesrepublik Deutschland}

Das erste Beispiel ist Deutschland als ein Bundesstaat vom Typ des kooperativen Föderalismus. ${ }^{6}$

Solidarität: Die deutsche Fiskalpolitik weist verschiedene Elemente der Solidarität auf. Das erste Element ist die bundesweit festgelegte Steueraufteilung zwischen dem Bund und den Ländern mit eingebautem, an der Angleichung der Lebensverhältnisse orientiertem Transfermechanismus zwischen den 16 Bundesländern. Insgesamt beziehen die Bundesländer aus diesem System (im Jahr 2010) etwa 94 Prozent ihrer Haushaltseinnahmen. Nur etwa 6 Prozent stammen dagegen aus eigenen Landes-Steuern. ${ }^{7}$ Für sieben der 16 Länder kommen durch dieses System hohe Transfer-Gewinne zustande: für fünf ,neue Länder' sind das durchschnittlich 12 Prozent der Einnahmen, für Bremen 32 Prozent, für Berlin 43 Prozent und für die übrigen deutlich weniger. ${ }^{8}$ Vier Bundesländer finanzierten durch ihre TransferVerluste den horizontalen Länderausgleich. Dazu kommen zentral organisierte und finanzierte automatische Stabilisatoren. Das zweite Element ist die bundesstaatliche Garantie für die Rückzahlung der von den Ländern aufgenommenen Kredite. ${ }^{9}$

Kontrolle: Der weitreichenden Solidarität stand bis vor wenigen Jahren in der Bundesrepublik nur eine sehr schwache bundesstaatliche beziehungsweise horizontale Kontrolle der Länder-Fiskalpolitik gegenüber. Gegenüber zur Defizitausweitung entschlossenen Landesregierungen konnte sich diese schwache Kontrolle nicht durchsetzen. Bis zum Jahr 2009 galt also eine weitgehende finanzpolitische Autonomie der Bundesländer, ${ }^{10}$ die auch die Kreditaufnahme einschloss. Diese machten davon vor allem bei ihrer Ausgabengestaltung und bei der Verschuldung Gebrauch. ${ }^{11}$

\section{Vereinigte Staaten von Amerika}

Das zweite Beispiel sind die Vereinigten Staaten von Amerika, ebenfalls ein insgesamt erfolgreicher Bundesstaat (vom Typ des sogenannten Konkurrenz-Föderalismus).

Solidarität: Auch die USA haben in ihrem Fiskalföderalismus starke Elemente der Solidarität. Erstens gibt es ein starkes, allerdings nicht an regionaler Angleichung der Lebensverhältnisse orientiertes Transfersystem, das den ,states‘ im Jahr 2010 circa 20 Prozent ihrer jeweiligen Einnahmen bereitgestellt hat. ${ }^{12}$ Dazu gehören zentral organisierte und finanzierte

6 Besonders zu den hier folgenden Ausführungen stütze ich mich verschiedentlich auf Konrad/Zschäpitz: Schulden ohne Sühne, 2012.

7 2010er Zahlen für die Bundesrepublik in: Bundesfinanzministerium: Bund/Länder-Finanzbeziehungen auf der Grundlage der Finanzverfassung, Ausgabe 2011, Berlin, S. 23-24.

8 Ebenda, S. 58 und 66.

9 Konrad/Zschäpitz: Schulden ohne Sühne, 2012, S. 91-95.

10 Art. 109 Grundgesetz.

11 Das ergibt sich zum Beispiel aus Eckhard Wurzel: Towards more efficient government: Reforming federal fiscal relations in Germany, Organisation for Economic Co-operation and Development: OECD Working Paper ECO/ WKP(99)1, Paris 1999; vgl. auch zu den Ergebnissen der Föderalismuskommissionen in Deutschland: Beate Jochimsen/Kai Konrad (Hrsg.): Föderalismuskommission II: Neuordnung von Autonomie und Verantwortung, Frankfurt 2008.

12 U.S. Department of Commerce, Economics and Statistics Administration, U.S. Census Bureau: State and Local Government Finances Summary: 2010, Governments Division Briefs, September 2012. 
automatische Stabilisatoren. Hinzu kommen eigene Steuern und andere Einnahmen der , states', die etwa 80 Prozent der Haushalte finanzieren.

Kontrolle: Auch in den USA sind die ,states' finanzpolitisch autonom. Davon macht man in den USA weit stärker Gebrauch als in Deutschland, vor allem durch eine weitergehende autonome Steuergesetzgebung und Steuererhebung der ,states'. Dazu zählen zum Beispiel Mehrwertsteuern (,sales taxes ${ }^{6}$ ) und Einkommenssteuern. Auch die Kreditaufnahme der ,states` fällt darunter. Aber es gibt keine Bundesgarantie für die Rückzahlung der von ihnen aufgenommenen Kredite und der Bund weigert sich auch erfolgreich, überschuldeten oder sonst in Zahlungsschwierigkeiten geratenen, states ' finanziell zu helfen. Allenfalls geschieht das indirekt und teilweise, zum Beispiel durch öffentliche Infrastrukturinvestitions-Programme wie in der aktuellen Krise.

Ergebnis: Erstens muss man eine markante Ähnlichkeit betonen: In beiden Bundesstaaten bewahren sich die Gliedstaaten weitreichende finanzpolitische Autonomie. Diese Autonomie ist übrigens bei den meisten reifen Bundesstaaten gegeben. Sie ist gleichsam eines der entscheidenden Kriterien für das Bestehen von Bundesstaatlichkeit. Zweitens bestehen auch große Unterschiede: In der Summe existiert eine deutlich geringere fiskalische Solidarität des Bundes in den USA mit seinen Gliedstaaten: Die ,states ' haben eine weit höhere finanzpolitische Eigenverantwortung, auch mit Blick auf die Überwindung regionaler Entwicklungsunterschiede; und vor allem gibt es keine Bail-Out-Zusicherung.

Was heißt das Ergebnis nun bezüglich der zurzeit in der Eurozone oft geäußerten Vermutung, dass eine Verstärkung der Solidarität gegenüber höher verschuldeten Eurostaaten die Wahrscheinlichkeit erhöht, dass diese ihre Schulden bedienen, ,aus ihnen hinauswachsen' und höhere Wachstumspotenziale entwickeln werden?

In den Nettoempfängern unter den Ländern der Bundesrepublik sollte laut dieser These, angesichts der im Vergleich zu den USA deutlich höheren Solidaritäts-Leistungen, eigentlich eine nachhaltige Senkung der Kreditaufnahme und der Verschuldung, unter das in den USA herrschende Niveau eintreten und zu höherem Wachstum, Wettbewerbsfähigkeit und geringerem Transferbedarf führen. Die Realität zeigt aber ein anderes Bild.

In der Bundesrepublik verschlechtert sich die Haushaltslage der Netto-Solidaritätsempfänger unter den Bundesländern seit Jahrzehnten, bei vielen in dieser Gruppe mit einer ständig weiterlaufenden und sich noch verstärkenden Verschuldung bei unzureichendem Wachstum. Demgegenüber waren es nur die Nettozahler-Länder die trotz dieser zusätzlichen Ausgaben auch ihre Haushalte konsolidierten und in Konkurrenzfähigkeit und Wachstum ihrer Wirtschaft vorangehen. Die Verschuldung der deutschen Bundesländer variierte Ende 2010 zwischen den Extremwerten von etwa 10 und 65 Prozent des jeweiligen Bruttoinlandsprodukts (BIP). ${ }^{13}$ Die überschuldeten Länder erfordern eine permanente Haushalts-Nothilfe durch den Bund und andere Länder, zusätzlich zu den besonders reichlichen Transferleistungen, die dort eigentlich autonome Wachstums- und Entschuldungspotenziale stärken sollten.

Dabei verändern sich die Ratings aber nur sehr geringfügig zu Ungunsten der höher verschuldeten Länder und die ,spreads ${ }^{6}$ - die Differenzen der Kreditkosten - bleiben auf sehr geringem Niveau. Diese Länder zahlen fast ebenso wenig für ihre Kredite wie die anderen,

13 Simone Scharfe: Schulden des öffentlichen Gesamthaushaltes am 31. Dezember 2010, in: Statistisches Bundesamt (Hrsg.): Finanzen und Steuern, November 2011, S. 1117-1125, hier S. 1121. 
die besser wirtschaften und sich weniger verschulden. Sie haben also wenig Anreiz, sich selbst um eine Verbesserung ihrer Lage zu bemühen. ${ }^{14}$

Die Zahl der Nettoempfängerländer übersteigt sehr deutlich die Zahl der Länder, die die Transfers finanzieren. Diese Relation wurde durch die Wiedervereinigung noch maßgeblich gesteigert. Das führt dazu, dass es in Deutschland keine politischen Mehrheiten gibt, die eine Veränderung dieses Systems erlauben würden. Dafür bleibt den Nettozahlern nur der Gang zum Bundesverfassungsgericht.

Erst gegen Ende der zweiten Föderalismuskommission im Jahr 2009 gelang eine Änderung der Lage durch die Einrichtung von Schuldenbremsen auf Bundes- und Länderebene. ${ }^{15}$

In den USA, wo die harte No-Bailout-Linie des Zentralstaats und das niedrigere Solidaritäts- beziehungsweise Transferniveau eigentlich zu einer Verschlimmerung der Lage bei den Gliedstaaten führen sollte, findet sich eine weit geringere öffentliche Verschuldung die Spanne liegt zwischen circa 7 und 28 Prozent des BIP - und ein höherer Reformdruck seitens der Investoren. Im öffentlich bekanntesten Fall eines zu hoch verschuldeten ,state', Kalifornien, sind es nur 22 Prozent seines BIP. ${ }^{16}$ Trotzdem gehen die Ratings dort schon auf diesem niedrigen Verschuldungsniveau deutlich weiter auseinander als in Deutschland, ebenso die Kreditkosten. ${ }^{17}$ Kalifornien hat angesichts des Ausbleibens von Bundeshilfen aus Washington D.C. bereits überaus drastische Maßnahmen zur Budgetsanierung ergriffen.

\section{Kontrolle durch die Finanzmärkte in den USA stärker und wirksamer}

Der Vergleich macht deutlich, dass die zentralstaatliche Kontrolle der gliedstaatlichen Verschuldung durch die Finanzmärkte eine bedeutsame und wirksame Ergänzung erhält. Die Kontrolle durch die Finanzmärkte ist in den USA ungleich stärker als in der Bundesrepublik. Angesichts des Fehlens einer wirksamen zentralstaatlichen Kontrolle in beiden Bundesstaaten waren die Gliedstaaten der USA also insgesamt einer wirkungsvolleren Kontrolle ihres fiskalpolitischen Verhaltens unterworfen als die Bundesländer in Deutschland.

\section{Bailout-Verbote als fiskalpolitisches Disziplinierungsinstrument}

Wenn man die Ergebnisse dieser beiden unterschiedlichen Kombinationen von Elementen der Solidarität und der Eigenverantwortung in zwei reifen Bundesstaaten vergleicht und wenn man nach Erklärungen für das Ausbleiben der eigentlich erwarteten Ergebnisse sucht, dann wird man rasch diejenige Komponente der beiden Modelle finden, die eindeutig mit den Unterschieden in den Ergebnissen korreliert. Das ist der Grad fiskalpolitischer Eigen-

14 Kirsten H. Heppke-Falk/Guntram B. Wolff: Moral hazard and bail-out in fiscal federations: Evidence for the German Länder, in: Deutsche Bundesbank: Discussion Paper Series 1: Economic Studies Nr. 7/2007; Rolf Obertreis: Deutschland, einig Schuldenland, in: Badische Zeitung, 26.6.2012; Franz von den Driesch: Land statt Bund, in: Anleihen Monitor, 2.7.2012.

15 Vgl. dazu auch Christian Kastrop/Gisela Meister-Scheufelen/Margaretha Sudhof/Werner Ebert: Konzept und Herausforderungen der Schuldenbremse, in: Aus Politik und Zeitgeschichte 13/2012, S. 1-8.

16 Christopher Chantrill: Gross public debt in 2010, in percent of gdp, State and Local debt, in: abrufbar unter: http://www.usgovernmentspending.com/compare_state_spending_2010pH0c (letzter Zugriff: 14.12.2012). Nicht enthalten sind die teilweise hohen Verbindlichkeiten für die künftigen Altersbezüge der öffentlichen Angestellten.

17 Josh Barro: Evaluating States' Credit with Bond Yields, in: Manhattan Institute: Issue Brief 6/2010. Hier lagen 2010 die Spreads zwischen Bonds der am besten und am schlechtesten gerateten ,states ‘ auf der einen und vergleichbaren US Treasury Bonds auf der anderen Seite zwischen immerhin schon 0,74 und 2,2 Prozentpunkten. 
verantwortung der Gliedstaaten. Wie schon mehrfach angesprochen, gibt es hier einen wesentlichen Unterschied zwischen dem US-amerikanischen (sowie kanadischen und schweizerischen) Modell des Konkurrenzföderalismus einerseits und dem deutschen (und zum Beispiel österreichischen) Modell des kooperativen Föderalismus andererseits. Laut einer internationalen Vergleichsstudie der Organization for Economic Co-operation and Development (OECD) ist diese Eigenverantwortung im Konkurrenzföderalismus (USA) vergleichsweise viel höher als im kooperativen Föderalismus (Deutschland), am stärksten dadurch gekennzeichnet, dass der Zentralstaat keine Verantwortung für die von seinen Gliedstaaten aufgenommenen Kredite übernimmt. ${ }^{18}$

Dem Ergebnis des OECD-Vergleichs zufolge sind Gliedstaaten in Bundesstaaten mit einer derartigen Regelung deutlich erfolgreicher bei der Sicherstellung eines soliden Haushalts als Gliedstaaten für die der jeweilige Zentralstaat bürgt. Das bestätigt sich auch beim Blick auf unseren Vergleich zwischen Deutschland und den USA.

Zentralstaatliche Schuldengarantien und umfassende Transfersysteme für die Gliedstaaten gehen dagegen eher einher mit hohen sowie dauerhaften Gliedstaats-Verschuldungen und mit der Selbstverstärkung des Zwanges zu immer weiteren zentralstaatlichen Hilfen. ${ }^{19}$

Die volle Eigenhaftung der ,states' macht auch nachvollziehbar warum die Ratingagenturen die Gliedstaaten der USA kontinuierlicher und kritischer prüfen als die deutschen Bundesländer. Ebenso plausibel ist, dass die Finanzmärkte schon bei geringerer Verschuldung rascher und spürbarer auf die Bonitätsveränderungen der amerikanischen ,states ‘ reagieren.

Und genauso liegt es vor diesem Hintergrund nahe, dass die ,states ' bei einer Herabstufung und drohender Kreditverteuerung vergleichsweise rasch mit eigenen Sparmaßnahmen und Steuererhöhungen reagieren um den Schaden zu begrenzen.

Wie der Föderalismusforscher Jonathan Rodden aus Stanford vor zwei Jahren schrieb: „While states occasionally harbor hopes of explicit federal debt guarantees, as with California's quixotic request in 2009, the federal government routinely turns them down, and after some delay, states are left to fire school teachers and raise taxes all on their own." ${ }^{20}$ Rodden stellt auch fest, dass die ,states' (nicht Washington D.C.!) sich schon im Zuge dieser Erfahrungen seit dem 19. Jahrhundert nach und nach veranlasst sahen, das zu tun, was deutsche Länder erst seit 2009 und unter dem Druck des Bundes fertigbrachten, nämlich ausdrückliche Begrenzungen der Kreditaufnahme in ihre Verfassungen zu schreiben. ${ }^{21}$

Im kooperativen Föderalismus Deutschlands verursachen zentralstaatliche Schuldengarantien und reichliche Transfersysteme dagegen bei den Ländern ein Maß an ,moral hazard“ das entscheidend zu der seit den 1960er Jahren teilweise überbordenden und weiter anhaltenden Verschuldungsbereitschaft sowie zum Verzicht auf energische eigene Sanierungsbemühungen beigetragen hat. ${ }^{22}$

Entsprechend hat die Bundesregierung seit Beginn der 1990er Jahre und noch zusätzlich angestachelt durch die Stabilitätsvorgaben des EU-Stabilitäts- und Wachstumspakts im Zuge von zwei nationalen Föderalismuskommissionen Anläufe unternommen, um die Verschuldungstendenz von Bund und Ländern zu stoppen. Erst im Jahr 2009 ist es ihr gelungen, eine

18 Isabelle Joumard/Per Mathis Kongsrud: Fiscal Relations Across Government Levels, Organisation for Economic Co-operation and Development, Economics and Statistics Department: Working papers 375/2003.

19 Jonathan Rodden: Hamilton's Paradox: The Promise and Peril of Fiscal Federalism, New York 2006.

20 Jonathan Rodden: The Future of European Federalism: South America or North America?, im Erscheinen.

21 Ebenda und Bundesfinanzministerium: Die Schuldenbremse - Für die Zukunft unserer Kinder, 2010.

22 Heppke-Falk/Wolff: Moral hazard and bail-out in fiscal federations, 2007, Zusammenfassung. 
verfassungsmäßige Schuldenbremse zu schaffen, ${ }^{23}$ und auch die Höhe der künftigen Transferleistungen für verschuldete Länder ausdrücklich infrage zu stellen. ${ }^{24}$

\section{Staatsschuldenkrise in der Eurozone und bundesstaatliche Fiskalsysteme}

Vor dem Hintergrund des bisher Gesagten kann man sich nun der Staatsschuldenkrise in der Eurozone zuwenden. Bei näherer Betrachtung wird sehr schnell klar, dass beide Ebenen viel mehr gemeinsam haben als man in der politischen und wissenschaftlichen Diskussion wahrnimmt.

Schließlich liegt mit der Europäischen Währungsunion ein bundesstaatlich organisiertes Währungssystem vor und die fiskalpolitischen Interdependenzen der Eurostaaten untereinander sowie mit der Euro-Gruppe oder der Europäischen Zentralbank weisen sehr starke Analogien zum Fiskalföderalismus von reifen Bundesstaaten auf. Tatsächlich stellen sich grundlegende Fragen wie die nach Solidarität, Kontrolle und ,moral hazard“ in beiden Feldern in gleicher oder fast gleicher Weise.

Bei einer Prüfung ist allerdings noch einmal zu betonen, dass beide nationale fiskalische Systeme tatsächlich bundesstaatlich organisiert sind, während die Eurozone eine zentralistische Koordination aufweist, beziehungsweise noch weiter verstärkt. Die Eurostaaten unterwerfen sich in Bezug auf ihre Haushaltsgestaltung und vor allem ihre Kreditaufnahme einem Maß gegenseitiger Kontrolle und Sanktionsmöglichkeiten, die sehr viel weiter gehen, als es für die Gliedstaaten in den Bundesstaaten USA oder Deutschland gilt. Dafür sorgen das geltende Vertragsrecht sowie der Stabilitäts- und Wachstumspakt.

Zweitens haben die USA oder Deutschland als reife Bundesstaaten föderale Haushalte, die sehr viel größer sind als der Haushalt der Europäischen Union. Entsprechend können Bundesländer und ,states ' von den jeweiligen staatlichen Gesamteinnahmen nur einen deutlich geringeren Anteil für sich beanspruchen.

\section{Die zwei Euro-Systeme: 2000 bis 2009 und 2010 bis 2012}

Nach drei Jahren Krisenmanagement in der Eurozone muss man zwischen zwei EuroSystemen unterscheiden, demjenigen bis zum Jahr 2009 und dem danach. Im Folgenden wird vor dem Hintergrund der zuvor geschilderten Erfahrungen verglichen, welchem der beiden eben diskutierten so unterschiedlichen Fiskalföderalismus-Modelle diese beiden Euro-Systeme am stärksten ähneln. Dabei stehen wiederum die Variablen der Solidarität, der Kontrolle und des ,moral hazard“ im Vordergrund. Die Kontrolle wird hier aber von vornherein durch den Beitrag der Finanzmärkte ergänzt.

\section{Das Eurosystem bis 2009}

Solidarität: Ausdrücklich sollte die finanzpolitische Solidarität unter den Eurostaaten deutlich geringer sein als in der Bundesrepublik. Vor allem wollten die Regierungen der Mitgliedstaaten keine gemeinschaftliche Garantie der öffentlichen Schulden. Im Vergleich der beiden Modelle konnte man zu Beginn der Währungsunion eine klare Orientierung am amerikanischen Vorbild ausmachen. So entspricht die No-Bailout-Klausel des Vertrags über

23 Vgl. dazu auch Kastrop/Meister-Scheufelen/Sudhof/Ebert: Konzept und Herausforderungen der Schuldenbremse, 2012.

24 Vgl. ebenda. 
die Arbeitsweise der Europäischen Union ${ }^{25}$ dem Schlüsselelement des amerikanischen Modells und ist diesem möglicherweise auch abgeschaut.

Neben dieser Einschränkung stand allerdings - dem deutschen Modell entsprechend eine umfangreiche Unionssolidarität mit den wirtschaftlich schwächsten Mitgliedstaaten, die ihren Ausdruck in der Regional-, Kohäsions- und Agrarpolitik findet.

Kontrolle: Der Sinn der No-Bailout-Klausel war entsprechend, mit der Vorenthaltung von Garantien, die Akteure der Finanzmärkte als Mit-Kontrolleure für die nationalen Fiskalpolitiken in der entstehenden Währungsunion zu gewinnen. Die Stärkung von ,moral hazard‘Effekten in den Mitgliedstaaten der Eurozone, wie sie aufgrund der Euro-Einführung denkbar geworden und in Deutschland wohlbekannt waren, sollte damit verhindert werden.

Allerdings ergänzte der Vertrag die marktgesteuerte Kontrolle durch ein von den zentralen Unionsorganen anzuwendendes und mit Sanktionsrecht versehenes Kontrollsystem, den Stabilitäts- und Wachstumspakt. Dieser Pakt entspricht mit seiner Kombination von Normen und Verhandlungen mehr einer bundesdeutschen Tradition des Umgangs mit Konflikten im Föderalismus. ${ }^{26}$ Er war ähnlich schwach wie die deutsche innerstaatliche Koordinierung der Länder-Fiskalpolitiken.

Insofern gab es eine erkennbare Unentschlossenheit der Euro-Gruppe zwischen dem amerikanischen und dem deutschen Modell, welche Marktakteure wie Regierungen daran zweifeln ließ, dass man sich in der Praxis wirklich für eine konsequente Durchsetzung des einen oder des anderen Elements entscheiden können würde. Außerdem waren die No-BailoutKlausel und der Stabilitäts- und Wachstumspakt letztlich ,weich' und ließen Schlupflöcher offen.

Ergebnis: Beide Elemente der Kontrolle versagten im ersten Jahrzehnt des Euro. Die NoBailout-Klausel erlangte erst im Jahr 2010 unerwartet eine gewisse Glaubwürdigkeit. Die Sanktionsdrohung des Stabilitäts- und Wachstumspakts wurde ebenfalls nicht ernst genommen, zu recht wie sich seit 2002-2003 zeigte. Im Übrigen hat die Eurozone bereits mit dem - uneffektiven - Stabilitäts- und Wachstumspakt einen Grad zentraler Koordinierung der mitgliedstaatlichen Fiskalpolitik erreicht, der in den reifen Bundesstaaten, die sie als ihre Vorbilder sieht, nicht akzeptiert wird.

\section{Stabilitäts- und Wachstumspakt}

Die meisten Eurostaaten respektierten die Regeln des Stabilitäts- und Wachstumspakts zwar aus eigenem Interesse und passten ihre Struktur- und Fiskalpolitiken den neuen Vorgaben erfolgreich an. Die drei Großen (Deutschland, Frankreich, Italien), plus Portugal und Griechenland, zogen es aber vor, die Defizitgrenzen zu überschreiten und ihre Verschuldung zu erhöhen. Dafür gab es Gründe politischer und institutioneller Natur. So war bei den Großen im Vergleich zu den Kleinen der Anpassungsdruck weniger, das Zeit-Inkonsistenz-Problem dagegen stärker wirksam.

Sie blieben unsanktioniert. Dem lagen die schon erwähnten Schwächen des Stabilitätsund Wachstumspakts zugrunde, etwa die weitgehende Politisierung des Kontroll- und Sanktionsverfahrens zwischen den Vertretern der Regierungen der Eurostaaten. Sie verhinderten, dass im Falle von Verstößen gegen den Stabilitäts- und Wachstumspakt wirksame Sanktionen durchgesetzt werden konnten.

25 Art. 125 AEUV.

26 Vgl. Manfred G. Schmidt: Das politische System Deutschlands, München 2007, S. 212 und 236. 


\section{Die No-Bailout-Klausel}

Auch die No-Bailout-Klausel hat zur Verhinderung von ,moral hazard‘ bis zum Jahr 2009 nichts oder nur sehr wenig beigetragen, weil die Mehrheit der Akteure sie schon zu Beginn der Währungsunion nicht ernst nahm, dies gilt sowohl für die Experten wie auch für die Finanzmarktakteure. Das lag einmal am Wortlaut des Art. 125 AEUV, der zum Beispiel der Europäischen Zentralbank offenbar einen solchen ,Bailout' durch den Aufkauf von Staatsanleihen am Sekundärmarkt gestattete. ${ }^{27}$ Es lag auch daran, dass die meisten Kenner der Materie sich angesichts der Dominanz der Regierungsvertreter im Verfahren bei einem übermäßigen Defizit nicht vorstellen konnten, dass und wie die Euro-Gruppe eines ihrer Mitglieder in die Insolvenz gehen lassen würde. Über die Option der Insolvenz eines Mitgliedstaates schweigen sich die EU-Verträge und der Stabilitäts- und Wachstumspakt ohnehin vollständig aus; dort ist nur von finanziellen Sanktionen die Rede.

Ein früher Indikator für diese ,optimistische' Sicht war zum Beispiel die Verringerung der ,spreads ${ }^{6}$ zwischen deutschen und südeuropäischen Anleihezinsen in den Jahren nach 1994 im Vorfeld der Euroeinführung, die die Bundesregierung zu ihrem Ruf nach einem Stabilitätspakt veranlasste. ${ }^{28}$ In der Wahrnehmung der Finanzmarktakteure galt also bis 2009 zunehmend mehr das deutsche als das amerikanische Modell.

Die Finanzmärkte verteuerten die Kreditkosten der südeuropäischen Staaten nur marginal und diejenigen der Großen gar nicht. Im Hinblick auf Griechenland und auf Portugal, wohl auch auf Italien, verschlechterten sich die staatlichen Schuldentragfähigkeiten aber so, dass diese Kreditverteuerungen höher hätten ausfallen sollen. Man kann in dieser Phase im Falle der drei ,Großen', Griechenland und Portugal sowie für ihre Gläubiger bereits von ,moral hazard'sprechen, mit Blick auf die Staatsverschuldung von Irland und Spanien aber beispielsweise nicht. ${ }^{29}$

Dass das kritische Potenzial Griechenlands und anderer Staaten erkannt worden war, wurde dadurch deutlich, dass es seit Mitte der 2000er Jahre mehrfach Anläufe einzelner Regierungen von Eurostaaten gab, die angesichts der Inaktivität der Finanzmarktakteure wenigstens von der Europäischen Zentralbank verlangten, beim Ankauf von Anleihen dieser Staaten Preisabschläge vorzunehmen. Europäische Zentralbank und Europäische Kommission wiesen dieses Ansinnen wiederholt ausdrücklich und auch erfolgreich ab. ${ }^{30}$

Erst seit die Investoren ab 2009 doch noch ihren Glauben an die automatische und volle finanzielle Solidarität unter den Eurostaaten verloren und die sogenannten GIIPS-Staaten ${ }^{31}$ mit einer dramatischen Verschlechterung ihrer Ratings auch eine kaum finanzierbare Steigerung ihrer Kreditkosten erlebten, wurde der, moral hazard` dieser Staaten beziehungsweise ihrer Gläubiger beendet. Die Logik des amerikanischen Modells begann zu funktionieren bis hin zu einer Teilinsolvenz für Griechenland, aber erschreckend spät und destabilisierend.

27 So unter anderem Jürgen von Hagen: Fiscal Sustainability in EMU. From the Stability and Growth Pact To A Stability Council for EMU, Papier präsentiert beim CNB-CERGE-CSE seminar, 11.12.2003, S. 2; eine juristische Bewertung auf aktuellem Stand findet sich bei Müller-Graff: Editorial, 2012, S. 2.

28 Theo Waigel, Interview in: Focus 37/1995, S. 42-44.

29 Schlussendlich anderer Meinung ist Jens Boysen-Hogrefe. Er bezieht sich allerdings nur auf die dem 2008er Crash direkt vorangehende Phase, wo er keinen ,moral hazard' sieht. Vgl. Jens Boysen-Hogrefe: Für einen Schuldenschnitt und gegen den Rettungsschirm? Argumente auf dem Prüfstand, in: Institut für Weltwirtschaft: Kiel Policy Brief 29/2011.

30 Christian Deubner: A Dynamic Perspective for the Reform of the Stability and Growth Pact, Centre d'études prospectives et d'informations internationales: CEPII Working Paper 6/2006, S. 35-36; einer der ersten deutschen Anwälte dieser Form der Sanktionierung war Stefan Collignon: Geldwertstabilität für Europa, Gütersloh 1996, S. 134.

31 Griechenland, Irland, Italien, Portugal und Spanien. 
Insgesamt gab es allzu lange einen Gleichschritt fortgesetzter Solidarität und unzureichender Kontrolle mit einem in bedenklicher Weise entstehenden ,moral hazard`.

\section{Die entstehende Fiskalunion ab 2010}

Angesichts der Erfahrungen mit der fiskalpolitischen Kontrolle in der Eurozone hätte man sich vorstellen können, dass die Regierungen der Eurostaaten für die Governance-Reformen nach 2009 entschiedener als zuvor das Vorbild des US-amerikanischen (oder eines anderen konkurrenzföderalistischen) Modells herangezogen hätten. Dafür hätte auch seine weit größere Kompatibilität mit den Grundprinzipien des fiskalischen Föderalismus und mit den mitgliedstaatlichen Souveränitäten gesprochen. Die Plötzlichkeit und Übertriebenheit der Reaktionen der Finanzmärkte hätten auf diese Weise und mit zusätzlichen Maßnahmen unterdrückt werden können.

Tatsächlich richten sich die Reformen nach 2009 aber noch mehr nach dem deutschen Modell institutionell-verfahrensmäßiger Kontrolle plus fiskalpolitischer Solidarität.

Solidarität: Einerseits ist in die Eurozone in Reaktion auf den Schock von 2010 mit dem ESM nun auch eine starke Komponente fiskalpolitischer Solidarität eingebaut worden - für Fälle von Liquiditäts- oder Solvenzschwächen bei Mitgliedstaaten und auch Banken. Vorläufer dazu waren die umfangreichen konditionierten Kredite an die sogenannten ,Programmstaaten' Griechenland, Portugal und Irland, für die der EFSF eingesetzt wurde. ${ }^{32}$

Kontrolle: Andererseits wird auf Drängen Deutschlands und anderer Eurostaaten seit 2010 durch den Six-Pack und Fiskalpakt die institutionelle Koordinierung der nationalen Fiskalpolitiken in der Eurozone drastisch verschärft, um Überschuldungssituationen auf lange Sicht zu verhindern und das Vertrauen der Finanzmärkte in die Anleihen aller Eurostaaten wieder zu gewinnen. Insbesondere wird die Machtposition der Europäischen Kommission in dieser Koordinierung noch weiter aufgewertet und gestärkt. Die Handhabung der Konditionalität gegenüber den schon erwähnten ,Programmstaaten “ gibt einen Hinweis darauf, wie weit äußerstenfalls die Eingriffsrechte der Eurozone in die Souveränität ihrer Mitgliedsstaaten gehen können.

Die Kontrollfunktion der Finanzmärkte dagegen soll durch die neuen Solidaritätsinstrumente eingeschränkt werden. Diese sollen den Druck der Finanzmarktakteure auf die Krisenstaaten verringern.

Ergebnis: Im Ergebnis ist das amerikanische Modell nach 2010 also deutlich zurückgedrängt worden. ${ }^{33}$ Demgegenüber sind institutionelle und prozedurale Elemente, die man aus dem deutschen Fiskalföderalismus kennt, noch weiter in den Vordergrund getreten und geschärft worden. Die Unentschiedenheit zwischen beiden Ansätzen wäre damit zugunsten einer eher deutschen Lösung ausgeräumt.

32 Vgl. Bundesfinanzministerium: Euro-Schutzschirm, 2012.

33 Am deutlichsten sichtbar im verringerten ,private sector involvement' im ESM. In den ersten Entwürfen für einen ESM vom Herbst 2010 steht es noch ausdrücklich im Vertragstext. In der definitiven Vertragsfassung vom Februar 2012 findet es nur in der Präambel eine kurze Erwähnung. Siehe Allgemeine Merkmale des künftigen Mechanismus. Erklärung der Euro-Gruppe vom 28. November 2010, als Annex zu: Schlussfolgerungen der Staats- und Regierungschefs der Mitgliedstaaten des Euro-Währungsgebiets vom 11. März 2011, S. 13-15. Treaty establishing the European Stability Mechanism, Brüssel 2.2.2012, T/ESM 2012/en. Anders schien das noch Mitte November 2012 Charles Wyplosz zu sehen, der in einer Konferenz im Bundesfinanzministerium einen dringenden Appell für die Umorientierung des Eurosystems hin zum amerikanischen Modell vortrug. Vgl. Charles Wyplosz: Two Models for Fiscal Discipline, Vortrag anlässlich der Konferenz Shaping the Fiscal Institutions of Europe, 21.11.2012. 
Würde in einer ersten positiven Reaktion angenommen, dass dieses reformierte System tatsächlich vollständig umgesetzt und funktionieren wird, dann wäre in dieser Sicht die fiskalpolitische Solidarität deutlich gestärkt. Im Gegenzug würde aber auch die fiskalpolitische Disziplinierung so verschärft, dass der Einsatz dieser Solidarität weitgehend unnötig würde. ,Moral hazard' wäre wirksam eingeschränkt und eine langfristige Stabilisierung der Eurozone wäre auf Dauer denkbar.

Den maßgeblichen Äußerungen der deutschen Bundeskanzlerin und des deutschen Finanzministers zufolge ist dies die Strategie der Bundesregierung. ${ }^{34}$

Allerdings ist die Eurozone im Gegensatz zu den reifen Bundesstaaten, die viele als ihre Vorbilder sehen, mit der verschärften Koordinierung der mitgliedstaatlichen Fiskalpolitik jetzt einen großen Schritt in eine Richtung gegangen, die eher zu einem Zentralstaat als zu einem Bundesstaat passt. Die Europäische Union ist jedoch nicht einmal das Letztere. Sie kann allenfalls als Konföderation zwischen in vieler Hinsicht weiterhin souveränen Staaten gelten.

Aus diesem Grund ist auch eine negative Reaktion auf das reformierte System gerechtfertigt. In ihrem Licht erscheint die volle Durchsetzung und Funktionsfähigkeit des neuen zentralistischen Koordinierungsverfahrens auf mittlere Sicht als höchst zweifelhaft. Es ist zu komplex in seiner funktionalen Verknüpfung verschiedener nationaler und gemeinschaftlicher Institutionen und Verfahren. Es geht so weit in seinem kontrollierenden und korrigierenden Eingriff in die nationalen haushaltspolitischen Prozesse und parlamentarischen Prärogative und es schränkt die mitgliedstaatliche Souveränität derart weit ein, dass es in seiner Umsetzung zu schweren Dysfunktionalitäten und Konflikten kommen dürfte.

Außerdem erscheint die Vorsorge für künftig mögliche Liquiditätskrisen - mit einer Schlüsselrolle für den ESM - nicht wirklich stabil. Das System müsste angesichts des auf überschuldeten und nicht mehr schuldentragfähigen Mitgliedstaaten lastenden hohen Schuldendienstes sein aktivierbares Hilfsvolumen auf lange Sicht so dimensionieren, dass es zusätzlich auch erlaubt, neuen und unter Umständen mehrfachen Refinanzierungskrisen dieser Eurostaaten wirksam zu begegnen. Experten bezweifeln, dass das in ausreichendem Maße geschieht. ${ }^{35}$

So wirkt es überdimensioniert für künftige normale Zeiten, trotzdem aber immer noch unterdimensioniert zur Bekämpfung größerer Vertrauenskrisen gegenüber großen überschuldeten Mitgliedstaaten. Das könnte erneut zu einer kurzfristigen Ausweitung der Hilfsgarantien, zur Abmilderung ihrer Konditionen und zur Inanspruchnahme der Europäischen Zentralbank für letztlich fiskalische Zwecke führen.

Im Ergebnis wäre das eine Kombination von schwacher unzureichender Kontrolle mit großer und nach oben offener Solidarität, eine klare Einladung zu mehr ,moral hazard` auf mittlere Sicht.

\section{Elemente des , amerikanischen 'Konkurrenzföderalismus bleiben präsent}

Die von der Eurozone gewählte Reformstrategie hat wichtige Elemente des ,amerikanischen' Konkurrenzföderalismus trotzdem noch nicht verdrängt. Skeptiker und Opponenten der neuen Zentralisierung sowie auch einige Unterstützer machen geltend, dass diese Elemente die Tragfähigkeit des Systems kurz- und mittelfristig auch dann stützen könnten, wenn

34 Vgl. Merkel: Rede im Europäischen Parlament, 2012.

35 So unter anderen Adrian Blundell-Wignall: Solving the Financial and Sovereign Debt Crisis in Europe, in: Financial Market Trends - OECD Journal 2/2012, S. 3-4. 
die zentrale Koordinierung sich als nicht voll durchsetzbar erweisen sollte oder wenn überschuldete Eurostaaten doch wieder an Vertrauen verlieren würden.

$\mathrm{Zu}$ diesen Elementen gehört paradoxerweise der Fiskalpakt, obwohl er eine wichtige Komponente der zentralen Koordinierung ist. Sie wirkt auf die Regierungen der Eurostaaten und indirekt auf die Finanzmärkte: Sein zentraler Bestandteil ist eine je nationale Selbstverpflichtung auf Schuldenabbau und die Einhaltung von Verschuldungsgrenzen, quasi eine Europäisierung der deutschen Schuldenbremse, aber auch ihrer viel älteren Vorbilder in den US-amerikanischen Gliedstaaten. Es bleibt allerdings noch ungewiss, ob Regelungen, die in den USA unter dem Druck der Märkte eingeführt wurden und funktionieren, die Verschuldungspolitik von Regierungen wirklich auch dann disziplinieren, wenn dieser Druck, wie in der Eurozone, durch die Existenz eines Rettungsschirmes abgemildert und durch ein institutionelles Regelsystem überlagert wird.

Ein zweites Element sind die Unwägbarkeiten der Hilfe im Falle neuerlicher Vertrauenskrisen. Sie wirken direkt auf die Finanzmärkte und indirekt auf die Regierungen der Eurostaaten: In ihren genauen Dimensionen schwer abschätzbar, gehören zu diesen Elementen die Erinnerung der Marktakteure an den weitgehenden Schuldenschnitt für Griechenland und die Tatsache, dass der Zugang zu den neuen Hilfs-Garantieleistungen des Rettungsschirms nicht komplikationslos und quasi automatisch gewährt wird.

Die hier zu erwartenden Ungewissheiten und Reibungsverluste dürften Wirkung zeigen. Dazu tritt die schmerzhafte Konditionalität des Rettungsschirms mit ihrer abschreckenden Wirkung für die Schuldner und den schwer berechenbaren Folgen für deren Schuldentragfähigkeit. Schließlich kommt hinzu, dass ,,auch diese Konditionalität weiterhin die Schuldnerhaftung beinhaltet und wegen der Souveränität der Schuldner die Gläubigerhaftung nicht ausschaltet" .36

Insgesamt dürfte dieses Paket neuer und kritischer Erwartungen die Investoren tatsächlich dazu bringen, stärker als in der Vergangenheit auf die Bonität ihrer Schuldner zu schauen, und dementsprechend beim Anleihekauf auch Risikoprämien durchzusetzen.

In der Summe darf man in dieser zweiten Phase des Eurosystems also trotz befürchteter Schwächen der zentralistischen Kontrolle und der weiter ausgebauten Solidarität darauf hoffen, dass wenigstens vorerst der , moral hazard` bei Staaten und Investoren zurückgedrängt wird.

\section{Die Zweideutigkeit im neuen Euro-System kann nicht überleben. Die Eurozone braucht das amerikanische Modell}

Damit befände sich die Eurozone also einerseits noch immer ein Stück weit zwischen dem amerikanischen und dem deutschen beziehungsweise europäischen Modell. Andererseits haben die Eurostaaten sie inzwischen aber deutlicher zum deutschen Modell hin ausgerichtet. Die ,amerikanischen' Strukturelemente passen dazu noch weniger als in der Phase bis 2009 und dürften teilweise an Bedeutung verlieren.

Vor allem die Unwägbarkeiten der Hilfe im Falle neuerlicher Vertrauenskrisen dürften für viele Finanzmarktakteure unakzeptabel bleiben. Sie werden bei neuerlichen Fällen dieser Art auf eine weitere Reduzierung solcher Ungewissheiten drängen. In Bezug auf den ESM-Vertrag hat das offenbar bereits gewisse Erfolge gehabt. Damit bleiben auch die Regierungen der Eurostaaten noch auf sehr lange Sicht unter einer Bedrohung, die sie immer

36 Vgl. Boysen-Hogrefe: Für einen Schuldenschnitt, 2011, S. 8. 
wieder an den Punkt bringen kann, an dem sie sich zu einem noch weiter erleichterten Zugang zu Finanzhilfen oder direkt zur Vergemeinschaftung aller staatlichen Schulden, ohne Wenn und Aber gedrängt sehen.

Im Gesamtergebnis und auf kurze Sicht kann die derzeitige Mischung der beiden Modelle die Anreize zu einem disziplinierten fiskalpolitischen Verhalten von Regierungen und Finanzmarktakteuren und damit Vertrauensbildung und Stabilisierung noch begünstigen. Schon auf mittlere Sicht dürfte allerdings das Übergewicht des deutsch inspirierten institutionell-prozeduralen Ansatzes, zusammen mit dem Wunsch der meisten Regierungen der Eurostaaten nach mehr und möglichst unbegrenzter fiskalischer Solidarität, dafür sorgen, dass die Solidaritätskomponenten der reformierten Governance weiter gestärkt und die verbliebenen Elemente marktgesteuerter Koordinierung nach amerikanischem Modell weiter verringert werden. Die Dynamik, die Solidarität und gegenseitige Verantwortungsbereitschaft in der Eurozone entfalten werden, wird diesen Prozess beschleunigen. Tatsächlich ist eine effektive Koexistenz beider Modelle in einer Währungsunion kaum vorstellbar.

Wenn dann, wie zu befürchten ist, tatsächlich keine wirksame zentralisierte Fiskalkoordinierung in der ,Eurozone` entstanden sein würde, dürfte das spätestens mittelfristig und auf Dauer wieder ,moral hazard‘ begünstigen. Die deutsche Mitgliedschaft in der Eurozone könnte dann doch noch in grundsätzliche Gefahr kommen.

Deshalb sollte neben der aktuellen offiziellen Reformstrategie einer institutionell-prozedural funktionierenden Koordinierung plus mehr fiskalischer Solidarität für den Fall ihres Scheiterns eine Alternativstrategie vorbereitet werden. Sie kann nur die volle Übernahme des US-amerikanischen Modells mit einer maßgeblichen Rolle für die Finanzmarktakteure bei der fiskalpolitischen Koordinierung in der Eurozone bedeuten. 